\title{
EVALUATION OF SINGULAR INTEGRALS BY HYPERBOLIC TANGENT BASED TRANSFORMATIONS
}

\author{
BEONG IN Yun
}

\begin{abstract}
We employ a hyperbolic tangent function to construct nonlinear transformations which are useful in numerical evaluation of weakly singular integrals and Cauchy principal value integrals.

Results of numerical implementation based on the standard Gauss quadrature rule show that the present transformations are available for the singular integrals and, in some cases, give much better approximations compared with those of existing non-linear transformation methods.
\end{abstract}

\section{Introduction}

In implementation of a numerical method such as, for example, the boundary element method, accurate evaluation of weakly singular integrals and Cauchy principal value integrals is required. Lots of methods for numerical evaluation of those singular integrals have been developed. Among them we are interested in the non-linear coordinate transformation method known to be efficient and easy to use in adaptive approaches. The non-linear coordinate transformations can be classified as polynomial transformations $[1,2,10,12,15,16,19]$ and sigmoidal transformations $[3,4,5,6,7,8,9,11,13,17,20,22,23]$. In addition, there is the singularity substraction method [18] accompanied with a non-linear transformation and the composite transformation method [21] of a polynomial transformation and a sigmoidal transformation.

For weakly singular integrals, most non-linear coordinate transformations have the property of null Jacobian at the singularity, which weakens the order of the original singularity. In the literature [18] it was reported that the higher order Sato polynomial transformation [16] results in very accurate approximation as long as only end-point weakly singular integrals are concerned. On the other hand, sigmoidal transformations can be used for both interior-point

Received April 30, 2009.

2010 Mathematics Subject Classification. 65D30, 65D32, 41A55.

Key words and phrases. hyperbolic tangent, weakly singular integral, Cauchy principal value integral, non-linear transformation.

This research was supported by Basic Science Research program through the National Research Foundation of Korea(NRF) funded by the Ministry of Education, Science and Technology(20100003925). 
weakly singular integrals and end-point ones without splitting the integration interval [20]. Moreover, explicit estimates of the asymptotic truncation errors for the Gauss quadrature rule combined with the sigmoidal transformation were given by Johnston and Elliott [9].

For Cauchy principal value integrals, only some particular polynomial transformations are useful while all the sigmoidal transformations are available via the Euler-Maclaurin formula [5, 6]. In [21], it was shown that the combination of the fourth degree polynomial given by Doblaré and Gracia [2] and a sigmoidal transformation is superior to a polynomial transformation or a sigmoidal transformation used alone.

As far as the author is aware, however, any existing non-linear coordinate transformation method does not provide accurate approximation to a weakly singular integral whose order of singularity is near -1 and to a Cauchy principal value integral in which the location of singularity is very close to an end-point of the integration interval.

In the next section, we suggest two sigmoidal-like functions based on the hyperbolic tangent function. In Section 3, non-linear transformations composed of the functions developed in Section 2 are suggested for end-point weakly singular integrals and interior-point ones, respectively. Numerical examples in which the order $\alpha$ of the weak singularity is in the range of $-0.5 \leq \alpha<-1$ are included to show the availability of the present non-linear transformations combined with the standard Gauss quadrature rule. The present method appears to be particularly effective when the orders of singularities are near -1 . In Section 4, for Cauchy principal value integrals, we apply the hyperbolic tangent based function given in Section 2 to the composite transformation method [21]. Numerical results show the superiority of the present method in the case when singular points are close to an end-point of the integration interval.

\section{Functions based on the hyperbolic tangent}

Using the hyperbolic tangent, $\tanh x:=\left(e^{x}-e^{-x}\right) /\left(e^{x}+e^{-x}\right)$, we define a function

$$
\phi(\beta ; t):=\tanh \left[\frac{\beta}{2}\left(\frac{1}{1-t}-\frac{1}{1+t}\right)\right], \quad-1 \leq t \leq 1,
$$

for any $\beta>0$. It coincides with a so-called infinite order sigmoidal transformation [4] on $[-1,1]$ which was first introduced by Sag and Szekeres [14]. Its first derivative is

$$
\phi^{\prime}(\beta ; t)=\frac{\beta}{2}\left\{\frac{1}{(1-t)^{2}}+\frac{1}{(1+t)^{2}}\right\} \operatorname{sech}^{2}\left[\frac{\beta}{2}\left(\frac{1}{1-t}-\frac{1}{1+t}\right)\right]
$$

and an inverse of $\phi(\beta ; y),-1 \leq y \leq 1$, is

$$
\phi^{-1}(\beta ; y)=\frac{1}{A(\beta ; y)}\left\{-1+\sqrt{1+A(\beta ; y)^{2}}\right\}, A(\beta ; y)=\frac{2}{\beta} \tanh ^{-1}(y)
$$


We note that, for any integer $k \geq 1$,

$$
\frac{d^{k}}{d t^{k}} \phi\left(\beta ; \tau_{0}\right)=0
$$

at both end-points $\tau_{0}= \pm 1$. That is, all the derivatives of any order are zero at the end-points independently of the parameter $\beta$. In addition, the slope of a curve of $\phi(\beta ; t)$ at the midpoint is

$$
\phi^{\prime}(\beta ; 0)=\beta .
$$

Availability of $\phi(\beta ; t)$ for weakly singular integrals is investigated in the next section.

On the other hand, for any $\beta>0$, we suggest another hyperbolic tangent based function

$$
\psi(\beta ; t):=\frac{\tanh (\beta t)}{\tanh \beta}, \quad-1 \leq t \leq 1
$$

with a derivative

$$
\psi^{\prime}(\beta ; t)=\frac{\beta}{\tanh \beta} \operatorname{sech}^{2}(\beta t)
$$

and an inverse,

$$
\psi^{-1}(\beta ; y)=\frac{1}{\beta} \tanh ^{-1}(y \tanh \beta), \quad-1 \leq y \leq 1 .
$$

Particularly, $\psi(\beta ; t)$ converges to the linear map, $t$ as $\beta$ goes to 0 . We can readily derive the following properties.

Lemma 2.1. For any $\beta>0$, the function $\psi(\beta ; t)$ satisfies

(1) $\psi(\beta ; t) \in C^{\infty}[-1,1]$ and $\psi(\beta ; \tau)=\tau$ for some points $\tau=-1,0,1$.

(2) $\psi(\beta ; t)$ is strictly increasing on $[-1,1]$.

(3) $\psi^{\prime}(\beta ; t)$ is strictly increasing on $[-1,0]$ with $\psi^{\prime \prime}(\beta ; 0)=0$.

(4) For any integer $k \geq 1, \frac{d^{k}}{d t^{k}} \psi(\beta ; \pm 1)=O\left(\beta^{k} e^{-2 \beta}\right)$ and $\psi^{\prime}(\beta ; 0)=$ $O(\beta)$ as $\beta \rightarrow \infty$.

Figure 1 shows the graphs of the function $\psi(\beta ; t)$ and its derivative for each $\beta=0.01,2,4,8$. It can be seen that, from Lemma 2.1 and Figure $1, \psi(\beta ; t)$ possesses most features of the usual sigmoidal transformation extended to the interval $[-1,1]$. Moreover, referring to Lemma 2.1(4) and the infinite order sigmoidal transformation $\phi(\beta ; t)$ in $(2.1)$, we may regard $\psi(\beta ; \xi)$, with $\beta$ large enough, as an asymptotically infinite order sigmoidal transformation.

It will be shown that the function $\psi(\beta ; t)$ is very useful for evaluating the Cauchy principal value integral in Section 4. 


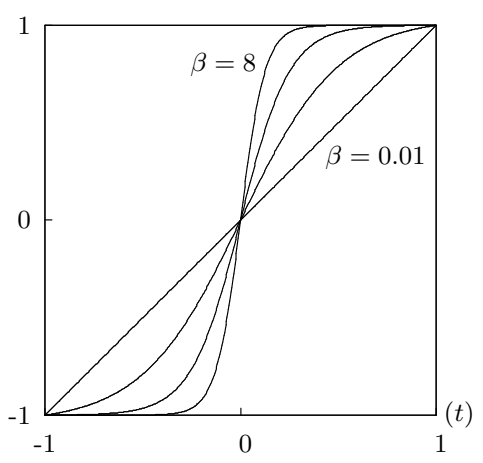

(a) $\psi(\beta, t)$

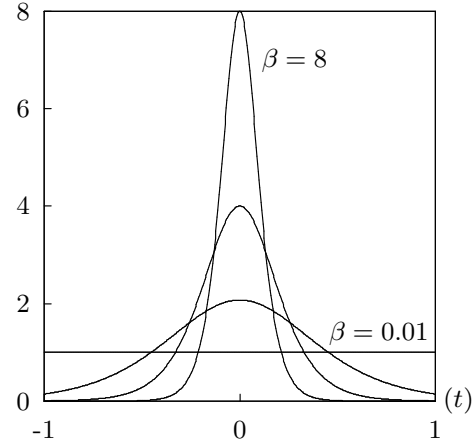

(b) $\psi^{\prime}(\beta, t)$

Figure 1. Graphs of the function $\psi(\beta ; t)$ and its derivative $\psi^{\prime}(\beta ; t)$ for each $\beta=0.01,2,4,8$.

\section{Weakly singular integrals}

\subsection{End-point singularities}

For the case of an end-point weak singularity in the integral over an interval $[-1,1]$, similarly to the semi-sigmoidal transformation $[8]$, we set a transformation based on the present function $\phi(\beta ; t)$ as

$$
\Phi(\beta ; t):=2 \phi\left(\beta ; \frac{t+\tau_{0}}{2}\right)-\tau_{0}, \quad-1 \leq t \leq 1
$$

for a singular point $\tau_{0}$ such that $\left|\tau_{0}\right|=1$. From the properties of the prototype function $\phi(\beta ; t)$ mentioned in the previous section, we can see that $\Phi(\beta ; \pm 1)=$ \pm 1 and $\Phi^{(k)}\left(\beta ; \tau_{0}\right)=0$ for all integers $k \geq 1$.

Suppose $\tau_{0}=1$ and a function $f(x)$ behaves like

$$
f(x) \sim c \cdot(1-x)^{\alpha}, \quad \alpha>-1,
$$

for $x$ near the end-point $\tau_{0}$. Then, by the change of variables $x=\Phi(\beta ; t)=$ $2 \phi\left(\beta ; \frac{t+1}{2}\right)-1$, we have

$$
\int_{-1}^{1} f(x) d x=\int_{-1}^{1} F(\beta ; t) d t,
$$

where $F(\beta ; t)$ is a transformed integrand defined by

$$
F(\beta ; t)=f(\Phi(\beta ; t)) \Phi^{\prime}(\beta ; t) .
$$

Theorem 3.1. For any $\alpha>-1$ and $\beta>0, F(\beta ; t)$ in $(3.4)$ has the asymptotic behavior

$$
F(\beta ; t)=O\left(\frac{1}{(1-t)^{2}} e^{-2(\alpha+1) \beta /(1-t)}\right)
$$

for $t$ near $\tau_{0}=1$. 
Proof. For $t \sim 1$, from (2.1) and (2.2),

$$
1-\phi\left(\beta ; \frac{t+1}{2}\right) \sim 1-\tanh \left[\frac{\beta}{1-t}\right]
$$

and

$$
\begin{aligned}
\phi^{\prime}\left(\beta ; \frac{t+1}{2}\right) & \sim \frac{2 \beta}{(1-t)^{2}} \operatorname{sech}^{2}\left[\frac{\beta}{1-t}\right] \\
& \sim \frac{4 \beta}{(1-t)^{2}}\left\{1-\tanh \left[\frac{\beta}{1-t}\right]\right\}
\end{aligned}
$$

Thus, from (3.1), (3.2) and (3.4), we have for $t \sim 1$

$$
\begin{aligned}
F(\beta ; t) & \sim c 2^{\alpha}\left\{1-\phi\left(\beta ; \frac{t+1}{2}\right)\right\}^{\alpha} \phi^{\prime}\left(\beta ; \frac{t+1}{2}\right) \\
& \sim c 2^{\alpha+2} \frac{\beta}{(1-t)^{2}}\left\{1-\tanh \left[\frac{\beta}{1-t}\right]\right\}^{\alpha+1} \\
& =c 2^{2 \alpha+3} \frac{\beta}{(1-t)^{2}}\left\{\frac{1}{e^{2 \beta /(1-t)}+1}\right\}^{\alpha+1} .
\end{aligned}
$$

Since $\alpha>-1$ and $\beta>0$, we have the asymptotic formula (3.5).

In addition, for any integer $k \geq 0$, it follows that

$$
\frac{d^{k}}{d t^{k}} F(\beta ; t)=O\left(\frac{1}{(1-t)^{2+2 k}} e^{-2(\alpha+1) \beta /(1-t)}\right)
$$

near $\tau_{0}=1$. This implies that the original weakly singular integrand $f(x)$ is transformed to an infinitely smooth one $F(\beta ; t)$ by the transformation $\Phi(\beta ; t)$ in (3.1)

\subsection{Interior-point singularities}

Suppose that a function $f(x)$ has a weak singularity like

$$
f(x) \sim c \cdot\left|x-s_{0}\right|^{\alpha}, \quad \alpha>-1,
$$

at an interior-point $-1<s_{0}<1$. We recall the generalized polynomial transformation (GPT) proposed by Monegato and Sloan [12], which does not require splitting the integration interval, in the form of

$$
\omega_{m}^{\mathrm{MS}}(t):=s_{0}+\delta\left(s_{0}, m\right) \cdot\left(t-t_{0}\right)^{m}, \quad-1 \leq t \leq 1,
$$

where

$$
\begin{aligned}
\delta\left(s_{0}, m\right) & =2^{-m}\left\{\left(1+s_{0}\right)^{1 / m}+\left(1-s_{0}\right)^{1 / m}\right\}^{m}, \\
t_{0} & =\frac{\left(1+s_{0}\right)^{1 / m}-\left(1-s_{0}\right)^{1 / m}}{\left(1+s_{0}\right)^{1 / m}+\left(1-s_{0}\right)^{1 / m}}
\end{aligned}
$$


and the order $m$ should be an odd integer. Another transformation method without splitting the integration interval, so-called the generalized sigmoidal transformation (GST), was introduced in [20] as below.

$$
\widetilde{\gamma}_{m}(t)=s_{0}+2 \operatorname{sgn}\left(t-t_{0}\right) \gamma_{m}\left(\frac{\left|t-t_{0}\right|}{2}\right), \quad-1 \leq t \leq 1,
$$

in which $\gamma_{m}(\xi), 0 \leq \xi \leq 1$, is a usual sigmoidal transformation of order $m \geq 2$. It was shown that this method can highly improve the accuracy of the standard Gauss quadrature rule for evaluating integrals with a weakly singular integrand such as $f(x)$ in (3.7).

Replacing $\gamma_{m}(\xi)$ in the generalized sigmoidal transformation $(3.9)$ by $\gamma(\xi)=$ $\frac{1}{2}\{\phi(2 \xi-1)+1\}$, we have

$$
\widetilde{\Phi}(\beta ; t):=s_{0}+\operatorname{sgn}\left(t-t_{0}\right)\left\{\phi\left(\beta ;\left|t-t_{0}\right|-1\right)+1\right\}, \quad-1 \leq t \leq 1,
$$

where $t_{0}=\phi^{-1}\left(\beta ; s_{0}\right)$ can be derived from (2.3), analytically. It follows that $\widetilde{\Phi}(\beta ; t)$ is strictly increasing with $\widetilde{\Phi}(\beta ; \pm 1)= \pm 1, \widetilde{\Phi}\left(\beta ; t_{0}\right)=s_{0}$, and since

$$
\widetilde{\Phi}^{\prime}(\beta ; t)=\phi^{\prime}\left(\beta ;\left|t-t_{0}\right|-1\right)
$$

from $(2.2), \widetilde{\Phi}^{\prime}\left(\beta ; t_{0}\right)=0$. Moreover, from $(2.4), \widetilde{\Phi}^{(k)}\left(\beta ; t_{0}\right)=0$ for all integers $k \geq 1$, which implies that $\widetilde{\Phi}(\beta ; t) \in C^{\infty}[-1,1]$. In addition, we can directly find that the first derivative $\widetilde{\Phi}^{\prime}(\beta ; t)$ has a maximum value $\beta$ at $t=t_{0}-\operatorname{sgn}\left(t_{0}\right)$. Figure 2 shows the graphs of the transformation $\widetilde{\Phi}(\beta ; t)$ and its derivative according to various values of $\beta$, from which one can grasp the feature of $\widetilde{\Phi}(\beta ; t)$ mentioned above.

If we consider an integral

$$
\int_{-1}^{1} f(x) d x=\int_{-1}^{1} F(\beta ; t) d t
$$

where $f(x)$ is a function having a weak singularity as (3.7) and

$$
F(\beta ; t)=f(\widetilde{\Phi}(\beta ; t)) \widetilde{\Phi}^{\prime}(\beta ; t),
$$

then, like the case of end-point singularity (Theorem 3.1), one can see that for any $t$ near $t_{0}$

$$
\frac{d^{k}}{d t^{k}} F(\beta ; t)=O\left(\frac{1}{\left|t-t_{0}\right|^{2+2 k}} e^{-2(\alpha+1) \beta /\left|t-t_{0}\right|}\right), \quad k \geq 0 .
$$

Therefore, the weak singularity of $O\left(\left|x-s_{0}\right|^{\alpha}\right)$ in the original integrand $f(x)$ is completely removed via the present transformation $\widetilde{\Phi}(\beta ; t)$ in $(3.10)$.

\subsection{Numerical examples}

For numerical examples we take an end-point weakly singular integral,

$$
I_{\alpha}:=\int_{-1}^{1}(1-x)^{\alpha} d x=\frac{2^{1+\alpha}}{1+\alpha}
$$




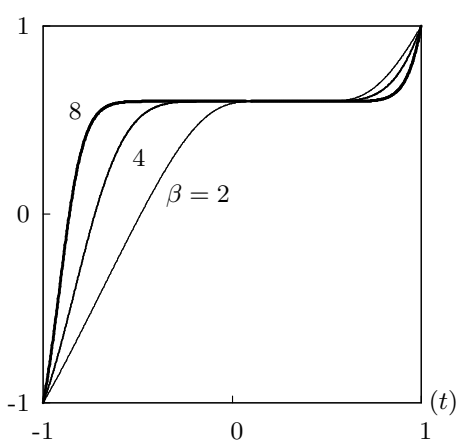

(a) $\tilde{\Phi}(\beta ; t)$

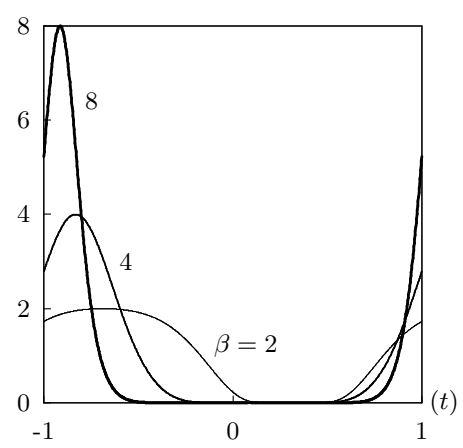

(b) $\tilde{\Phi}^{\prime}(\beta ; t)$

Figure 2. Graphs of the transformation $\tilde{\Phi}(\beta ; t)$ and its derivative $\tilde{\Phi}^{\prime}(\beta ; t)$ with $s_{0}=0.6$ for each $\beta=2,4,8$.

and an interior-point weakly singular integral,

$$
J_{\alpha}\left(s_{0}\right):=\int_{-1}^{1}\left|x-s_{0}\right|^{\alpha} d x=\frac{1}{1+\alpha}\left\{\left(1+s_{0}\right)^{1+\alpha}+\left(1-s_{0}\right)^{1+\alpha}\right\},
$$

where $-1<s_{0}<1$.

The integrals in the form of (3.13) or (3.14) with $0<\alpha<1$ can be approximated sufficiently accurately by any one of the traditional non-linear transformation methods. Thus, in this section, we consider the higher weak singular integrals and employ the standard Gauss quadrature rule in the numerical experiment.

First, for the integral $I_{\alpha}$ with $\alpha=-0.7,-0.9,-0.95$ numerical results of the existing non-linear transformation methods and the present method are included in Table 1. Therein, the Sato-polynomial transformation [16] denoted by $\omega_{m}^{\text {Sato }}$ is defined by

$$
\omega_{m}^{\text {Sato }}(t)=1-\frac{(1-t)^{m}}{2^{m-1}}, \quad-1 \leq t \leq 1
$$

and $\omega_{m}^{\text {semi }}$ is the semi-sigmoidal transformation [8] defined by

$$
\omega_{m}^{\text {semi }}(t)=1-4 \gamma_{m}\left(\frac{1-t}{4}\right), \quad-1 \leq t \leq 1
$$

where $\gamma_{m}(x)$ is the simple sigmoidal transformation [13] as

$$
\gamma_{m}(x)=\frac{x^{m}}{x^{m}+(1-x)^{m}}, \quad 0 \leq x \leq 1 .
$$

Noting that the slopes of the transformations at the opposite side of the singular point $t=1$ are

$$
\left(\omega_{m}^{\text {Sato }}\right)^{\prime}(-1)=\left(\omega_{m}^{\text {semi }}\right)^{\prime}(-1)=m, \quad \Phi^{\prime}(\beta ;-1)=\beta,
$$


we compare these transformations with $m=\beta$ in the numerical implementation. From Table 1 we can see that the superiority of present transformation $\Phi(\beta ; t)$ over $\omega_{m}^{\text {Sato }}(t)$ and $\omega_{m}^{\text {semi }}(t)$ becomes more distinct as the order of singularity $\alpha$ goes to -1 . In addition, for the case of $\alpha=-0.95$, Figure 3 compares relative errors of the transformations $\omega_{m}^{\text {Sato }}(t), \omega_{m}^{\text {semi }}(t)$ and $\Phi(\beta ; t)$ with $m=\beta=3(2) 25$. The accuracy of the transformation $\Phi(\beta ; t)$ obviously exceeds the Sato-polynomial transformation and the semi-sigmoidal transformation.

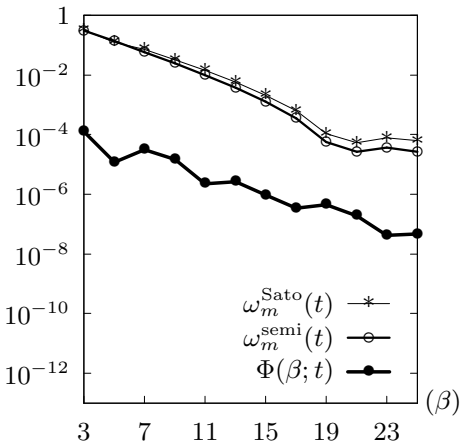

(a) $N=20$

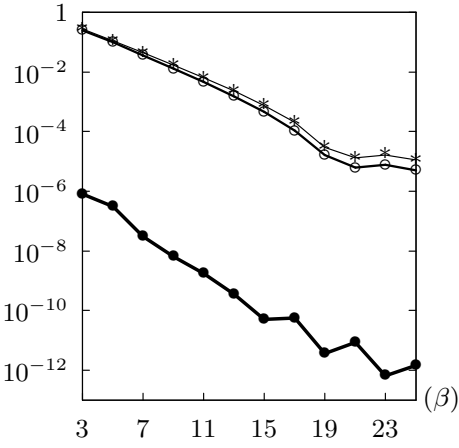

(b) $N=40$

Figure 3. Relative errors of the transformations $\omega_{m}^{\text {Sato }}(t), \omega_{m}^{\text {semi }}(t)$, and $\Phi(\beta ; t)$ for the weakly singular integral $I_{-0.95}$ with respect to $m=\beta=3(2) 25$.

For the interior-point weakly singular integral $J_{\alpha}\left(s_{0}\right)$ with $s_{0}=0$ in (3.14), numerical results of the GPT $\omega_{m}^{\mathrm{MS}}(x)$ in (3.8), the GST $\widetilde{\gamma}_{m}(t)$ in (3.9) and the present transformation $\widetilde{\Phi}(\beta ; t)$ in $(3.10)$ are compared in Table 2. For each case of $\alpha=-0.7,-0.9,-0.95$, the accuracy of $\widetilde{\Phi}(\beta ; t)$ is better than that of the others for $N \geq 20$.

\section{Cauchy principal value integrals}

\subsection{The composite transformation method}

For a function having a Cauchy singularity such as

$$
f(x)=\frac{g(x)}{x-s_{0}}, \quad-1 \leq x \leq 1,
$$

where $\left|s_{0}\right|<1$ and $g(x)$ is a well-behaved function with $g\left(s_{0}\right) \neq 0$, we refer to the composite transformation method [21] :

$$
\Psi_{m}(t):=\eta\left(1-2 \gamma_{m}\left(\frac{1-t}{2}\right)\right), \quad-1 \leq t \leq 1,
$$

where $\gamma_{m}$ is a sigmoidal transformation of order $m \geq 2$ and $\eta(t)$ is the fourth degree polynomial transformation introduced by Doblaré and Gracia [2] as

$$
\eta(t)=s_{0}\left(1-t^{4}\right)+t^{3}, \quad-1 \leq t \leq 1,
$$


TABLE 1. Relative errors of the transformations $\omega_{m}^{\text {Sato }}(t)$, $\omega_{m}^{\text {semi }}(t)$, and $\Phi(\beta ; t)$ with $m=\beta=9$ for the end-point weakly singular integral $I_{\alpha}$ in (3.13).

\begin{tabular}{ccccc}
\hline$\alpha$ & $N$ & $\omega_{m}^{\text {Sato }}(t)$ & $\omega_{m}^{\text {semi }}(t)$ & $\Phi(\beta ; t)$ \\
\hline \multirow{4}{*}{0.7} & 10 & $6.1 \times 10^{-7}$ & $6.9 \times 10^{-6}$ & $1.1 \times 10^{-5}$ \\
& 20 & $1.6 \times 10^{-8}$ & $2.9 \times 10^{-9}$ & $5.8 \times 10^{-10}$ \\
& 30 & $1.9 \times 10^{-9}$ & $3.5 \times 10^{-10}$ & $2.7 \times 10^{-13}$ \\
& 40 & $4.1 \times 10^{-10}$ & $7.7 \times 10^{-11}$ & $4.3 \times 10^{-16}$ \\
& 50 & $1.2 \times 10^{-10}$ & $2.3 \times 10^{-11}$ & $1.0 \times 10^{-16}$ \\
& 10 & $1.0 \times 10^{-3}$ & $5.8 \times 10^{-4}$ & $5.7 \times 10^{-5}$ \\
& & & & \\
-0.9 & 20 & $3.0 \times 10^{-4}$ & $1.7 \times 10^{-4}$ & $4.9 \times 10^{-7}$ \\
& 30 & $1.5 \times 10^{-4}$ & $8.4 \times 10^{-5}$ & $3.1 \times 10^{-9}$ \\
& 40 & $8.8 \times 10^{-5}$ & $5.0 \times 10^{-5}$ & $2.3 \times 10^{-11}$ \\
& 50 & $5.9 \times 10^{-5}$ & $3.4 \times 10^{-5}$ & $2.4 \times 10^{-13}$ \\
& & & & \\
& 10 & $5.9 \times 10^{-2}$ & $4.5 \times 10^{-2}$ & $1.5 \times 10^{-3}$ \\
& 20 & $3.2 \times 10^{-2}$ & $2.4 \times 10^{-2}$ & $1.5 \times 10^{-5}$ \\
0.95 & 30 & $2.3 \times 10^{-2}$ & $1.7 \times 10^{-2}$ & $2.6 \times 10^{-7}$ \\
& 40 & $1.7 \times 10^{-2}$ & $1.3 \times 10^{-2}$ & $6.4 \times 10^{-9}$ \\
& 50 & $1.4 \times 10^{-2}$ & $1.1 \times 10^{-2}$ & $2.7 \times 10^{-11}$ \\
\hline
\end{tabular}

TABLE 2. Relative errors of the transformations $\omega_{m}^{\mathrm{MS}}(t)$, $\widetilde{\gamma}_{m}(t)$, and $\widetilde{\Phi}(\beta ; t)$ with $m=\beta=9$ for the interior-point weakly singular integral $J_{\alpha}(0)$ in $(3.14)$.

\begin{tabular}{ccccc}
\hline$\alpha$ & $N$ & $\omega_{m}^{\mathrm{MS}}(t)$ & $\widetilde{\gamma}_{m}(t)$ & $\widetilde{\Phi}(\beta ; t)$ \\
\hline \multirow{4}{*}{0.7} & 10 & $9.1 \times 10^{-4}$ & $1.4 \times 10^{-4}$ & $7.7 \times 10^{-4}$ \\
& 20 & $1.5 \times 10^{-4}$ & $2.1 \times 10^{-5}$ & $8.4 \times 10^{-6}$ \\
& 30 & $5.1 \times 10^{-5}$ & $7.9 \times 10^{-6}$ & $4.2 \times 10^{-7}$ \\
& 40 & $2.4 \times 10^{-5}$ & $3.9 \times 10^{-6}$ & $8.5 \times 10^{-9}$ \\
& 50 & $1.3 \times 10^{-5}$ & $2.2 \times 10^{-6}$ & $2.7 \times 10^{-9}$ \\
& 10 & $1.3 \times 10^{-2}$ & $5.1 \times 10^{-3}$ & $2.5 \times 10^{-2}$ \\
& 20 & $7.2 \times 10^{-3}$ & $3.5 \times 10^{-3}$ & $1.1 \times 10^{-3}$ \\
-0.9 & 30 & $5.0 \times 10^{-3}$ & $2.6 \times 10^{-3}$ & $3.6 \times 10^{-4}$ \\
& 40 & $3.9 \times 10^{-3}$ & $2.1 \times 10^{-3}$ & $6.6 \times 10^{-5}$ \\
& 50 & $3.2 \times 10^{-3}$ & $1.7 \times 10^{-3}$ & $2.2 \times 10^{-5}$ \\
& & & & \\
& 10 & $2.0 \times 10^{-1}$ & $1.5 \times 10^{-1}$ & $8.6 \times 10^{-2}$ \\
& 20 & $1.5 \times 10^{-1}$ & $1.1 \times 10^{-1}$ & $2.0 \times 10^{-2}$ \\
0.95 & 30 & $1.3 \times 10^{-1}$ & $9.5 \times 10^{-2}$ & $5.1 \times 10^{-3}$ \\
& 40 & $1.1 \times 10^{-1}$ & $8.4 \times 10^{-2}$ & $7.5 \times 10^{-4}$ \\
& 50 & $1.0 \times 10^{-1}$ & $7.6 \times 10^{-2}$ & $8.7 \times 10^{-4}$ \\
\hline
\end{tabular}

satisfying $\eta(0)=s_{0}, \eta(-1)=-1, \eta(1)=1$, and $\eta^{\prime}(0)=\eta^{\prime \prime}(0)=0$. It was shown that the composite transformation method combined with the Gauss 
quadrature rule is efficient in the evaluation of Cauchy singular integrals for overall location of the singular point $-1<s_{0}<1$.

In this section, taking the composite transformation method above, we employ the function $\psi(\beta ; t)$ defined in $(2.6)$ instead of the sigmoidal transformation. That is, we set

$$
\Psi(\beta ; t):=\eta \circ \psi(\beta ; t)=\eta(\tanh (\beta t) / \tanh \beta), \quad-1 \leq t \leq 1 .
$$

From the properties of $\psi(\beta ; t)$ discussed in Section 2 , it follows that for any $\beta>0$

(i) $\Psi(\beta ; 0)=s_{0}, \Psi(\beta ;-1)=-1, \Psi(\beta ; 1)=1$,

(ii) $\Psi^{\prime}(\beta ; 0)=\Psi^{\prime \prime}(\beta ; 0)=0$.

Additionally, it can be seen that $\Psi(\beta ; t)$ converges to $\eta(t)$ as $\beta \rightarrow 0$. Figure 4 shows the graphs of $\Psi(\beta ; t)$ and its derivative for some values of $\beta$. It should be noted that, as $\beta$ becomes larger, the flatness of the curve near the midpoint $t=0$ is diffused to the end-points. Therefore, by change of variables $x=$ $\Psi(\beta ; t)$ in the integration of $f(x)$ as investigated below, the contribution of the transformed integrand $h(\beta ; t)=f(\Psi(\beta ; t)) \Psi^{\prime}(\beta ; t)$ to the numerical evaluation is concentrated in the vicinity of the midpoint as $\beta$ is increasing.

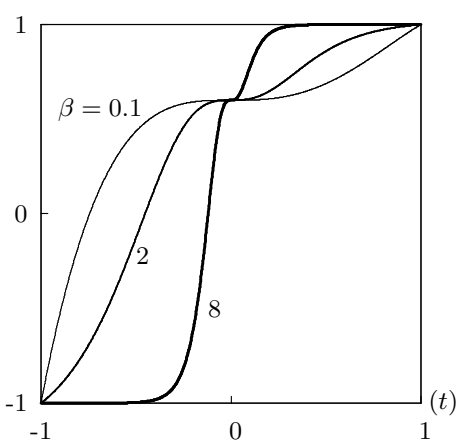

(a) $\Psi(\beta ; t)$

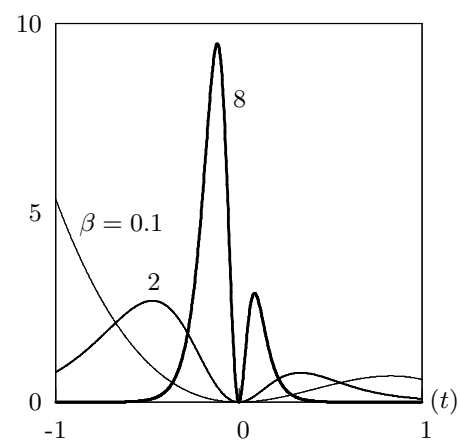

(b) $\Psi^{\prime}(\beta ; t)$

Figure 4. Graphs of the transformation $\Psi(\beta ; t)$ and its derivative $\Psi^{\prime}(\beta ; t)$ with $s_{0}=0.6$ for each $\beta=0.1,2,8$.

Now, for the function $f(x)$ given in (4.1), we consider the Cauchy principal value integral

$$
\begin{aligned}
\int_{-1}^{1} f(x) d x & =\int_{-1}^{1} \frac{g(x)-g\left(s_{0}\right)}{x-s_{0}} d x+g\left(s_{0}\right) \int_{-1}^{1} \frac{1}{x-s_{0}} d x \\
& :=K_{1}\left(s_{0}\right)+g\left(s_{0}\right) K_{2}\left(s_{0}\right) .
\end{aligned}
$$


Referring to the literature [2] and the properties (i) and (ii), we may notice that, by change of variables as $x=\Psi(\beta ; t)$, both the proper integral

$$
K_{1}\left(s_{0}\right)=\int_{-1}^{1} \frac{g(\Psi(\beta ; t))-g(\Psi(\beta ; 0))}{\Psi(\beta ; t)-\Psi(\beta ; 0)} \Psi^{\prime}(\beta ; t) d t
$$

and the Cauchy principal value integral

$$
K_{2}\left(s_{0}\right)=\int_{-1}^{1} \frac{\Psi^{\prime}(\beta ; t)}{\Psi(\beta ; t)-\Psi(\beta ; 0)} d t
$$

can be computed effectively by the standard Gauss quadrature rule with an even number of integration points.

It should be noted that, when the singular point $s_{0}$ in (4.5) is very close to an end-point, the quadrature rule combined with the polynomial transformation $\eta(t)$ results in poor accuracy [21]. Though the composite transformation $\Psi_{m}(t)$, in (4.2), combined with a special sigmoidal transformation such as $\gamma_{m}^{\mathrm{Y}}(b ; t)$ given in [23] can greatly improve the accuracy, it has disadvantages that the formula of $\gamma_{m}^{\mathrm{Y}}(b ; t)$ is rather complicated and that an adjustable procedure to find reasonable values of the parameter $b$ according to the location of $s_{0}$ is not known. However, in the following numerical example, we show that the presented composite transformation $\Psi(\beta ; t)$ in $(4.4)$ can overcome those problems.

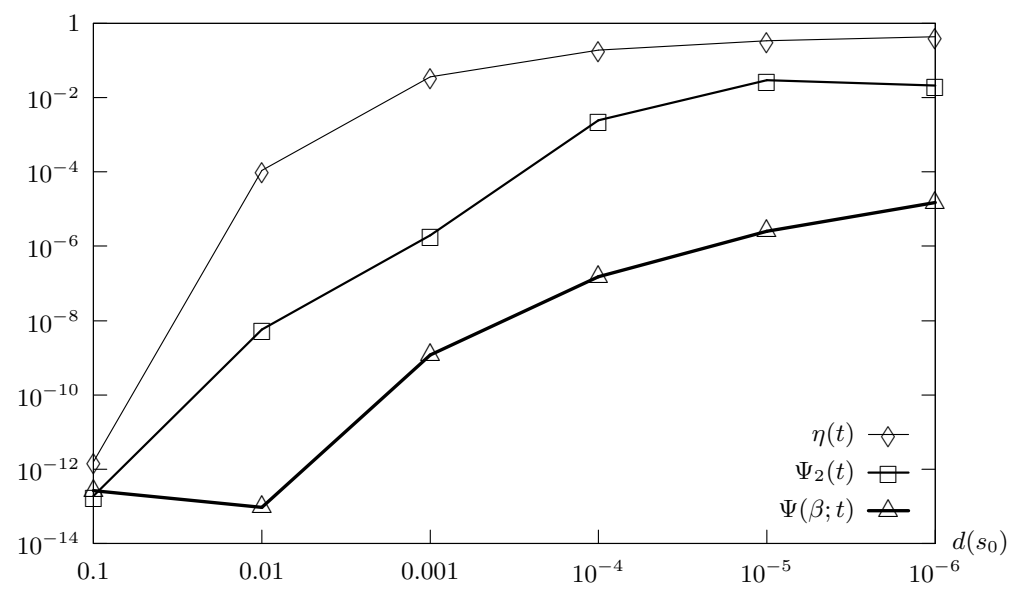

Figure 5. Relative errors of the transformations $\eta(t), \Psi_{2}(t)$, and $\Psi(\beta ; t)$, with $\beta=\beta\left(s_{0}\right)$ as given in $(4.7)$, for $K\left(s_{0}\right)$ with $d\left(s_{0}\right)=1-s_{0} \leq 0.1$.

\subsection{Numerical examples}

We consider a simple form of the Cauchy principal value integral

$$
K\left(s_{0}\right):=\int_{-1}^{1} \frac{1}{x-s_{0}} d x
$$


whose exact value is $\log \left(\frac{1-s_{0}}{1+s_{0}}\right)$.

In [21] it was mentioned that the lowest order $m=2$ of the composite transformation $\Psi_{m}(t)$ in (4.2) is reasonable to escape unnecessary clustering of the integration points near the singular point. Therefore, in this work, we compare numerical results of the proposed transformation $\Psi(\beta ; t)$ with those of the fourth degree polynomial transformation $\eta(t)$ and the composite transformation $\Psi_{2}(t)$ combined with the Sidi-sigmoidal transformation $\gamma_{2}(t)=(1-\cos \pi x) / 2$ as taken for examples in the literature [21]. In using the transformation $\Psi(\beta ; t)$, we suggest a formula to take $\beta=\beta\left(s_{0}\right)$ depending on the location of the singular point as follows:

$$
\beta=-2 \log _{10}\left[d\left(s_{0}\right)\right], \quad d\left(s_{0}\right)=\min \left\{1-s_{0}, 1+s_{0}\right\}
$$

which is increasing as the singular point $s_{0}$ comes closer to an end-point of the integration interval. In particular, when $s_{0}=0, \beta=0$ so that $\Psi(\beta ; t)$ coincides with $\eta(t)$.

For the Cauchy principal value integral $K\left(s_{0}\right)$ in (4.6), numerical results of the $N$ points Gauss quadrature rule combined with the transformations $\eta(t)$, $\Psi_{2}(t)$ and $\Psi(\beta ; t)$ are given in Table 3 . Additionally, Figure 5 shows the relative errors for near end-point singularities, in the range of $0.9 \leq s_{0} \leq 0.999999$, with $N=32$. That is, the distance between the singular point and an end-point $x=1$ is within $0.1 \leq d\left(s_{0}\right)=1-s_{0} \leq 10^{-6}$. In both Table 3 and Figure 5 , the presented transformation $\Psi(\beta ; t)$ takes values of $\beta$ as given in the formula (4.7).

\section{Conclusions}

In this paper, we have developed several hyperbolic tangent based transformations for accurate numerical evaluation of singular integrals using the standard Gauss quadrature rule. They can be summarized as follows:

1. For end-point weakly singular integrals: $\Phi(\beta ; t)$ in $(3.1)$ based on the function $\phi(\beta ; t)$ in $(2.1)$ and the semi-sigmoidal transformation method [8].

2. For interior-point weakly singular integrals: $\widetilde{\Phi}(\beta ; t)$ in $(3.10)$ based on the function $\phi(\beta ; t)$ and the generalized sigmoidal transformation method [20].

3. For Cauchy principal value integrals: $\Psi(\beta ; t)$ in $(4.4)$ based on the function $\psi(\beta ; t)$ in $(2.6)$ and the composite transformation method [21].

Numerical results for some examples of evaluating singular integrals have shown the superiority of the proposed methods over existing non-linear transformation methods. Moreover, it has been observed that the methods provide very accurate approximations especially for higher order weakly singular integrals and Cauchy principal value integrals having singular points which are very close to an end-point of the integration interval. 
TABLE 3. Relative errors of the transformations $\eta(t), \Psi_{2}(t)$ and $\Psi(\beta ; t)$ for the Cauchy principal value integral $K\left(s_{0}\right)$ in $(4.6)$.

\begin{tabular}{|c|c|c|c|c|c|}
\hline$s_{0}$ & $N$ & $\eta(t)$ & $\Psi_{2}(t)$ & $\Psi(\beta ; t)$ & $\beta$ in $(4.7)$ \\
\hline \multirow{5}{*}{0.2} & 4 & $1.6 \times 10^{-8}$ & $2.5 \times 10^{-4}$ & $3.3 \times 10^{-11}$ & \multirow{5}{*}{0.19382} \\
\hline & 8 & $7.9 \times 10^{-15}$ & $2.8 \times 10^{-9}$ & $2.3 \times 10^{-14}$ & \\
\hline & 12 & $2.3 \times 10^{-14}$ & $1.7 \times 10^{-13}$ & $2.2 \times 10^{-13}$ & \\
\hline & 16 & $2.7 \times 10^{-14}$ & $3.1 \times 10^{-14}$ & $3.6 \times 10^{-14}$ & \\
\hline & 20 & $1.4 \times 10^{-12}$ & $3.3 \times 10^{-13}$ & $1.1 \times 10^{-13}$ & \\
\hline \multirow{5}{*}{0.5} & 4 & $3.8 \times 10^{-5}$ & $5.1 \times 10^{-4}$ & $1.0 \times 10^{-7}$ & \multirow{5}{*}{0.60206} \\
\hline & 8 & $1.0 \times 10^{-9}$ & $1.5 \times 10^{-7}$ & $8.7 \times 10^{-15}$ & \\
\hline & 12 & $4.1 \times 10^{-14}$ & $2.2 \times 10^{-10}$ & $1.1 \times 10^{-14}$ & \\
\hline & 16 & $5.1 \times 10^{-13}$ & $2.1 \times 10^{-13}$ & $1.8 \times 10^{-13}$ & \\
\hline & 20 & $6.2 \times 10^{-13}$ & $5.1 \times 10^{-14}$ & $2.2 \times 10^{-13}$ & \\
\hline \multirow{5}{*}{0.8} & 4 & $5.1 \times 10^{-3}$ & $9.5 \times 10^{-3}$ & $5.4 \times 10^{-5}$ & \multirow{5}{*}{1.39794} \\
\hline & 8 & $2.1 \times 10^{-5}$ & $8.0 \times 10^{-6}$ & $1.7 \times 10^{-8}$ & \\
\hline & 12 & $8.2 \times 10^{-8}$ & $1.9 \times 10^{-7}$ & $1.3 \times 10^{-12}$ & \\
\hline & 16 & $3.2 \times 10^{-10}$ & $4.8 \times 10^{-10}$ & $1.2 \times 10^{-14}$ & \\
\hline & 20 & $2.3 \times 10^{-12}$ & $1.6 \times 10^{-12}$ & $6.4 \times 10^{-14}$ & \\
\hline \multirow{5}{*}{0.9} & 4 & $2.8 \times 10^{-2}$ & $6.6 \times 10^{-4}$ & $7.3 \times 10^{-4}$ & \multirow{5}{*}{2 . } \\
\hline & 8 & $7.1 \times 10^{-4}$ & $2.9 \times 10^{-4}$ & $1.6 \times 10^{-6}$ & \\
\hline & 12 & $1.7 \times 10^{-5}$ & $2.8 \times 10^{-6}$ & $6.3 \times 10^{-10}$ & \\
\hline & 16 & $4.2 \times 10^{-7}$ & $9.8 \times 10^{-9}$ & $9.2 \times 10^{-13}$ & \\
\hline & 20 & $1.0 \times 10^{-8}$ & $4.8 \times 10^{-10}$ & $4.9 \times 10^{-14}$ & \\
\hline
\end{tabular}

\section{References}

[1] M. Cerrolaza and E. Alarcón, A bi-cubic transformation for the numerical evaluation of the Cauchy principal value integrals in boundary methods, Internat. J. Numer. Methods Engrg. 28 (1989), no. 5, 987-999.

[2] M. Doblaré and L. Gracia, On non-linear transformations for the integration of weaklysingular and Cauchy principal value integrals, Internat. J. Numer. Methods Engrg. 40 (1997), no. 18, 3325-3358.

[3] D. Elliott, The cruciform crack problem and sigmoidal transformations, Math. Methods Appl. Sci. 20 (1997), no. 2, 121-132.

[4] Sigmoidal transformations and the trapezoidal rule, J. Austral. Math. Soc. Ser. B 40 (1998/99), (E), E77-E137.

[5] _ The Euler-Maclaurin formula revisited, J. Austral. Math. Soc. Ser. B 40 (1998/99), (E), E27-E76.

[6] D. Elliott and E. Venturino, Sigmoidal transformations and the Euler-Maclaurin expansion for evaluating certain Hadamard finite-part integrals, Numer. Math. 77 (1997), no. $4,453-465$

[7] P. R. Johnston, Application of sigmoidal transformations to weakly singular and nearsingular boundary element integrals, Internat. J. Numer. Methods Engrg. 45 (1999), no. 10, 1333-1348.

[8] _ Semi-sigmoidal transformations for evaluating weakly singular boundary element integrals, Internat. J. Numer. Methods Engrg. 47 (2000), no. 10, 1709-1730. 
[9] P. R. Johnston and D. Elliott, Error estimation of quadrature rules for evaluating singular integrals in boundary element problems, Internat. J. Numer. Methods Engrg. 48 (2000), no. 7, 949-962.

[10] _ A generalisation of Telles' method for evaluating weakly singular boundary element integrals, J. Comput. Appl. Math. 131 (2001), no. 1-2, 223-241.

[11] N. M. Korobov, Number-Theoretic Methods in Approximate Analysis, Gosudarstv. Izdat. Fiz.-Mat. Lit., Moscow, 1963.

[12] G. Monegato and I. H. Sloan, Numerical solution of the generalized airfoil equation for an airfoil with a flap, SIAM J. Numer. Anal. 34 (1997), no. 6, 2288-2305.

[13] S. Prössdorf and A. Rathsfeld, On an integral equation of the first kind arising from a cruciform crack problem, Integral equations and inverse problems (Varna, 1989), 210219, Pitman Res. Notes Math. Ser., 235, Longman Sci. Tech., Harlow, 1991.

[14] T. W. Sag and G. Szekeres, Numerical evaluation of high-dimensional integrals, Math. Comput. 18 (1964), 245-253.

[15] J. Sanz Serna, M. Doblaré, and E. Alarcón, Remarks on methods for the computation of boundary-element integrals by co-ordinate transformation, Comm. Appl. Numer. Methods 6 (1990), no. 2, 121-123.

[16] M. Sato, S. Yoshiyoka, K. Tsukui, and R. Yuuki, Accurate numerical integration of singular kernels in the two-dimensional boundary element method, in: C.A. Brebbia(Eds.), Boundary Elements X Vol.1, Springer, Berlin, 1988, pp. 279-296.

[17] A. Sidi, A new variable transformation for numerical integration, Numerical integration, IV (Oberwolfach, 1992), 359-373, Internat. Ser. Numer. Math., 112, Birkhäuser, Basel, 1993.

[18] K. M. Singh and M. Tanaka, On non-linear transformations for accurate numerical evaluation of weakly singular boundary integrals, Internat. J. Numer. Methods Engrg. 50 (2001), no. 8, 2007-2030.

[19] J. C. F. Telles, A self-adaptive co-ordinate transformation for efficient numerical evaluation of general boundary element integrals, Internat. J. Numer. Meth. Eng. 24 (1987), 959-973.

[20] B. I. Yun, An extended sigmoidal transformation technique for evaluating weakly singular integrals without splitting the integration interval, SIAM J. Sci. Comput. 25 (2003), no. 1, 284-301.

[21] _ A compositie transformation for numerical integration of singular integrals in the BEM, Internat. J. Numer. Methods Engrg. 57 (2003), no. 13, 1883-1898.

[22] _ A generalized non-linear transformation for evaluating singular integrals, Internat. J. Numer. Methods Engrg. 65 (2006), no. 12, 1947-1969.

[23] B. I. Yun and P. Kim, A new sigmoidal transformation for weakly singular integrals in the boundary element method, SIAM J. Sci. Comput. 24 (2003), no. 4, 1203-1217.

Department of InFormatics And Statistics

Kunsan National University

GUNSAN 573-701, KOREA

E-mail address: biyun@kunsan.ac.kr 